\title{
Is Brazilian music getting more predictable? A statistical physics approach for different music
}

genres

\section{Paulo Ferreira}

VALORIZA - Research Center for Endogenous Resource Valorization, Portalegre, Portugal Instituto Politécnico de Portalegre, Portalegre, Portugal

CEFAGE-UE, IIFA, Universidade de Évora, Largo dos Colegiais 2, 7000 Évora, Portugal

Email: pferreira@ipportalegre.pt

Telephone: (+351) 268628528

\section{Derick Quintino}

Department of Economics, Administration and Sociology, University of São Paulo, 13418-900 Piracicaba, SP,

$$
\text { e-mail: derickdq@usp.br }
$$

Bruna Wundervald

Hamilton Institute \& Department of Mathematics and Statistics,

Maynooth University, Maynooth, Kildare,

Ireland,

Email: bruna.wundervald@mu.ie

Andreia Dionísio

CEFAGE-UE, IIFA, Universidade de Évora, Largo dos Colegiais 2, 7000 Évora, Portugal

\section{Faheem Aslam}

Management Sciences, Comsats University, Islamabad 45550, Pakistan

Ana Cantarinha

Instituto Politécnico de Beja,

CIMA-Center for Research on Mathematics and its Applications, University of Évora; CMA-Center of Mathematics and Applications, FCTNOVA, NOVA University of Lisbon 


\section{Abstract}

Music is an important part of most people's lives and also of the culture of a country. Moreover, the different characteristics of songs, such as genre and the chord sequences, could have different impacts on individual behaviours. Even considering just seven chords and the respective variations, originality can be a crucial element of a song's success. Considering this, and in the context of Brazilian music, we employed the Detrended Fluctuation Analysis to analyse the possible predictability of eight different music genres. On these genres, we found that Reggae and Pop seem to be the least random considering the sequenced use of chords. With a sliding windows approach, we found that the predictability of chord sequences of Pop decreased over time. Applying the same methodology after shuffling the original series of music, the results point to a randomness of those shuffled series, demonstrating the robustness of our approach.

Keywords: Detrended Fluctuation Analysis; music genres; predictability; shuffle.

Acknowledgments: Paulo Ferreira acknowledges the financial support of Fundação para a Ciência e a Tecnologia (grants UIDB/05064/2020 and UIDB/04007/2020). Andreia Dionísio is pleased to acknowledge financial support from Fundação para a Ciência e a Tecnologia (grant UIDB/04007/2020). This study was financed in part by the Coordenação de Aperfeiçoamento de Pessoal de Nível Superior - Brasil (CAPES) FinanceCode 001. Derick Quintino wishes to acknowledge the CAPES for funding support. Bruna Wundervald acknowledges the financial support of Science Foundation Ireland through the Career Development Award grant number: $17 / \mathrm{CDA} / 4695$. 